necessitating the use of contrast enema and laparotomy, both of which increase the risks of necrotizing enterocolitis (NEC) and intestinal perforation. The need for surgical intervention and anaesthesia has a potential negative impact on the infant's future neurodevelopment.

Objectives Our pilot trial demonstrated that twice-daily saline enema is a feasible intervention to reduce time to reach full enteral feeds in very low birth weight (VLBW) infants. This study aims to explore the efficacy of saline enema in the treatment of MOP with respect to feasibility and safety by secondary analysis of data from the primary study which was a prospective open-label randomized controlled trial. We hypothesized that twice daily saline enema is an effective, feasible and safe intervention to treat MOP in VLBW infants as compared to glycerin suppositories (control).

Methods In this prospective open-label, pilot, randomized controlled trial, 61 infants who failed to pass meconium within 48 hours of birth were randomized to receive either twice-daily normal saline enema (SE) or a GS until they reached full enteral feeds $(110 \mathrm{~mL} / \mathrm{kg} / \mathrm{day}) .28$ were randomized to receive twice daily saline enemas (intervention) and 33 were controls who were treated conventionally with a glycerin suppositories as a control (GS, 2,000 mg, a quarter unit, 4 doses $12 \mathrm{~h}$ apart). A standardized feeding protocol and departmental guidelines for other aspects of neonatal intensive care were used in all subjects. The primary outcomes was treatment failure, defined as the need for an additional intervention. Adverse events (AE) such as colonic perforation and NEC were also recorded. We performed both Intention to Treat (ITT) and Per Protocol (PP) analyses. Data on efficacy, safety, process and resource feasibility outcomes were monitored.

Results Sixty-one infants were randomized, with 28 infants managed with RWs and 33 with glycerin suppositories. There was no treatment failure in the SE arm. Two infants were referred to surgeons, one with difficulty in passing the catheter and the other for blood-stained meconium. However, both infants were subsequently successfully managed with saline enema administered by pediatric surgeons. Two infants met the criteria for treatment failure in the GS arm. One infant was managed with contrast enema and the other was managed with SE administered by surgeons. Results from the ITT analysis did not differ substantially from the PP analysis.

Conclusions Analysis of outcomes of our study suggest that nurse administered twice daily saline enema is an efficacious, safe and cost-effective strategy for meconium obstruction of prematurity as compared to glycerin suppositories. Study design of future trials may be refined, to address the limitations of this study such as larger sample size, inclusion of infants with birth weight less than 750 grams, infants with atypical presentation and refinement of treatment failure criteria.

\section{DISRUPTION OF PAEDIATRIC ORTHOPAEDIC HOSPITAL SERVICES DUE TO THE COVID-19 PANDEMIC IN A REGION WITH MINIMAL COVID-19 ILLNESS}

Fui Lin Wong. UK

10.1136/bmjpo-2021-RCPCH.57
Background The Women's and Children's Hospital (WCH) in South Australia is a major tertiary referral teaching hospital with a Level 1 major paediatric trauma centre, receiving referrals from within South Australia and neighbouring states.

The setting for this study was unique as the hospital has no admitted cases of COVID-19 illness and, as such, this study represents the effects of government restrictions rather than the disease itself.

Objectives To evaluate the impact of the COVID-19 pandemic on paediatric orthopaedic services in a major paediatric tertiary hospital in South Australia.

Methods A retrospective audit was conducted of orthopaedic activity at a major paediatric tertiary referral hospital in South Australia, including a level 1 major paediatric trauma centre, for the period between 16 March 2020 and 26 April 2020 and compared with the corresponding time period in 2019 (18 March 2019 to 28 April 2019). Emergency Department (ED) presentations for musculoskeletal conditions, including fractures, were studied. Orthopaedic outpatient clinic, admission and operating theatre activity data were also assessed. No patients were admitted with COVID-19 illness at the hospital.

Results 621 paediatric patients presented to the ED with orthopaedic complaints during the 6-week period, compared to 997 in 2019, representing a 37.7\% reduction. However, there was minimal change in the number of ED presentations requiring hospital admission (110 in 2020 versus 116 in 2019). Among patients discharged directly from ED, $27.3 \%$ received hospital outpatient referral (versus 39.1\% in 2019), with the remaining patients referred to external health services including GP, mental health services or private heath providers) or discharged directly.

A total of 982 and 712 appointments (including face-toface and telehealth) were scheduled in 2019 and 2020 respectively (table 3). $20.6 \%$ of patients failed to attend their appointments in 2020, up from $14.5 \%$ in 2019. There was a $509.8 \%$ increase in telehealth (video and phone) outpatient consultations compared to 2019 and a $60.6 \%$ decline in faceto-face appointments.

There was a total of 144 orthopaedic admissions (elective and emergency) compared to 184 in 2019, representing a $21.7 \%$ decline. Elective admissions were halved from 68 in 2019 to 34 while emergency admissions reduced by $5.2 \%$ from 116 to 110 , demonstrating an overall shift in total workload towards emergency surgeries. Despite an overall downtrend in hospital admissions, elective and emergency admissions for children under 7 remained unchanged during the study period $(32.5 \%$ reduction in children aged 7 and above).

Conclusions Musculoskeletal problems are the sixth most common presentation in children and adolescents and a better understanding of the impact of this pandemic provides guidance for planning in the face of future pandemics, or a second wave of COVID-19.

Despite an overall decline in all paediatric orthopaedic hospital activities, the number of emergency admissions for musculoskeletal conditions did not change. Elective and emergency admissions for children under 7 remained unchanged. There is a significant uptrend in the use of outpatient telehealth services. Appropriate planning and hospital resources allocation are necessary to meet this service requirement in future pandemics. 


\section{Disruption of Paediatric Orthopaedic Hospital Services Due To The COVID-19 Pandemic In A Region with Minimal COVID-19 IIIness

\section{Introduction:}

This study evaluated the impact of the COVID-19 pandemic on paediatric orthopaedic services in the major paediatric tertiary hospital serving the state of South Australia and surrounding region. In contrast to other developed countries with advanced healthcare systems, South Australia has seen rapid control of COVID-19 cases and minimal mortality, despite initiating lockdown and restricting non-urgent elective surgeries in a similar timeframe. The setting for this study was unique as the hospital had no admitted cases of COVID-19 illness. As such, rather than the effects of the disease itself, this study represents the effects of government restrictions, the associated changes in local departmental policies and the change in patients' healthcare-seeking behaviour due to the pandemic. Musculoskeletal problems are the sixth most common presentation in children and adolescents and a better understanding of the impact of this pandemic provides guidance for planning of resource allocations in the face of future pandemics, or future waves of COVID-19.

\section{Methods}

A retrospective audit was conducted of orthopaedic activity at the major South Australian paediatric tertiary hospital, including a Level 1 paediatric trauma centre, serving a population of 1.8 million. The dates included were 16 March 2020 to 26 April 2020, corresponding to a time of statewide restrictions on non-essential movements and a legislated ban on all surgery except cases considered necessary to save life or prevent permanent disability. Emergency Department (ED) musculoskeletal presentations, orthopaedic outpatient clinics, surgeries and hospital admissions for the period between 16 March 2020 to 26 April 2020 were studied and compared with the corresponding period in 2019 (18 March 2019 to 28 April 2019).

\section{Results}

621 patients presented to the ED with orthopaedic complaints during the 6-week period in 2020 (versus 997 in 2019) (Table 1). However, there was minimal change in the number of ED presentations requiring admission (110 in 2020 versus 116 in 2019). Among patients discharged directly from ED, $27.3 \%$ received a hospital outpatient appointment (versus $39.1 \%$ in 2019), with the remaining patients referred to community health services or discharged directly.

$20.6 \%(147 / 565)$ of patients failed to attend their outpatient appointments in 2020 (versus $14.5 \%$ (142/840) in 2019) (Table 2). There was a $509.8 \%$ increase in telehealth (video and phone) outpatient consultations compared to 2019 and a $60.6 \%$ decline in face-to-face appointments.

There were 144 orthopaedic admissions (elective and emergency) compared to 184 in 2019, demonstrating a $21.7 \%$ decline. Elective admissions halved from the previous year while emergency admissions were only reduced by $5.2 \%$ Admissions for children under seven (elective and emergency) remained unchanged (versus $32.5 \%$ reduction in children aged seven and above) (Figure 3).

\section{Conclusion}

Despite an overall decline in paediatric orthopaedic hospital activity, the number of emergency admissions for musculoskeletal conditions did not change. Elective surgery numbers for children aged under seven were also unchanged, reflecting the urgency of many of these conditions. Appropriate planning and hospital resources allocation are necessary to meet this service requirement in future pandemics.

Article published in Journal of Children Orthopaedics, Vol 14, No. 4. Available from: https://doi.org/10.1302/1863-2548.14.200140
Table 1: Comparison of patient management after presentation to ED for the same time periods in 2019 and 2020.

\begin{tabular}{|lcc|c|}
\hline & 2019 & 2020 & p value \\
\hline ADMITTED & 116 & 110 & $<0.001$ \\
\hline DISCHARGED FROM ED & 877 & 510 & \\
\hline $\begin{array}{l}\text { FOLLOW-UP DESTINATION FROM ED } \\
\text { - Referral within WCH }\end{array}$ & $343(39.1 \%)$ & $139(27.3 \%)$ & \\
\hline $\begin{array}{l}\text { - External health providers } \\
\text { - Not referred }\end{array}$ & $281(32.0 \%)$ & $186(36.5 \%)$ & \\
\hline $\begin{array}{l}\text { LEFT BEFORE TREATMENT } \\
\text { COMPLETED }\end{array}$ & $253(28.8 \%)$ & $185(36.3 \%)$ & \\
\hline \begin{tabular}{l} 
TOTAL ED PRESENTATIONS \\
\hline
\end{tabular} & 4 & 1 & \\
\hline
\end{tabular}

Table 2: Comparison of outpatient clinic volumes and appointment types for the same time periods in 2019 and 2020.

\begin{tabular}{lcccc|}
\hline & 2019 & 2020 & Change (\%) & p value \\
\hline Total appointments & 982 & 712 & $-27.5 \%$ & \\
\hline $\begin{array}{l}\text { Attendance } \\
\text { - Attendance }\end{array}$ & 840 & 565 & -32.7 & $<0.001$ \\
- Non-attendance & 142 & 147 & +3.5 & \\
Modalities & & & & \\
- Face-to-face & 799 & 315 & -60.6 & $<0.001$ \\
\hline$-\quad$ Telehealth & 41 & 250 & +510 & \\
\hline
\end{tabular}

Figure 3: Comparison of elective and emergency admissions in children aged $<7$ years versus aged $\geq 7$ years in 2019 and 2020.

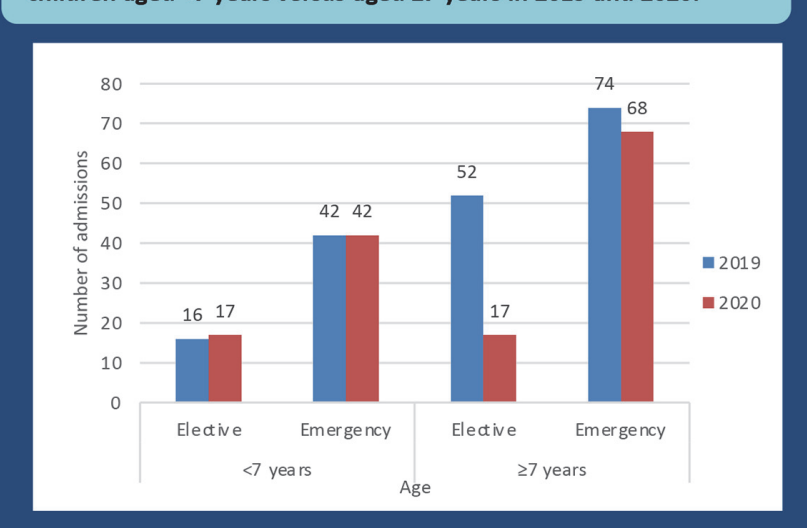

